\title{
Reversible Shaping of Microwells by Polarized Light Irradiation
}

\author{
Federica Pirani, ${ }^{1,2}$ Angelo Angelini, ${ }^{1}$ Francesca Frascella, ${ }^{1}$ and Emiliano Descrovi ${ }^{1}$ \\ ${ }^{1}$ Dipartimento di Scienza Applicata e Tecnologia, Politecnico di Torino, Corso Duca degli Abruzzi 24, 10129 Torino, Italy \\ ${ }^{2}$ Centro per le Tecnologie Future Sostenibili, Istituto Italiano di Tecnologia, Corso Trento 21, 10129 Torino, Italy \\ Correspondence should be addressed to Angelo Angelini; angelo.angelini@polito.it
}

Received 11 August 2017; Revised 12 October 2017; Accepted 26 October 2017; Published 3 December 2017

Academic Editor: Nobuhiro Kawatsuki

Copyright (C) 2017 Federica Pirani et al. This is an open access article distributed under the Creative Commons Attribution License, which permits unrestricted use, distribution, and reproduction in any medium, provided the original work is properly cited.

\begin{abstract}
In the last years, stimuli-responsive polymeric materials have attracted great interest, due to their low cost and ease of structuration over large areas combined with the possibility to actively manipulate their properties. In this work, we propose a polymeric pattern of soft-imprinted microwells containing azobenzene molecules. The shape of individual elements of the pattern can be controlled after fabrication by irradiation with properly polarized light. By taking advantage of the light responsivity of the azobenzene compound, we demonstrate the possibility to reversibly modulate a contraction-expansion of wells from an initial round shape to very narrow slits. We also show that the initial shape of the microconcavities can be restored by flipping the polarization by $90^{\circ}$. The possibility to reversibly control the final shape of individual elements of structured surfaces offers the opportunity to engineer surface properties dynamically, thus opening new perspectives for several applications.
\end{abstract}

\section{Introduction}

In recent years, the ability to pattern large areas of stimuliresponsive materials at the micro- and nanoscale has opened the opportunity to engineer surface structures and trigger peculiar properties such as complex optical functionalities [1] or surface properties [2] by light-matter interactions. Over the last two decades, the progress on micro- and nanoscale research has enabled the development of several patterning techniques, including soft lithography [3], nanoimprint lithography [4], interference lithography, and advanced photo lithography [5]. Micro- and nanofabrication technology finds applications in a plethora of fields, such as optics and photonics [6], microfluidics [7], and even biology [8].

However, despite such impressive achievements, most of the conventional patterning techniques result in static structures, whose features cannot be modified after fabrication. To overcome such limitation, Karageorgiev et al. proposed exploiting the photoresponsivity of azopolymers in a technique named Directional Photofluidization Lithography [911], although the term "photofluidization" is controversial as the fundamental mechanism underlying such effect is still under debate [12]. Based on the same approach, here we propose a reversible manipulation of prefabricated azopolymeric structures driven by polarized light.
Azopolymers are well known to transduce a visible light stimulus into a mechanical modification due to cyclic isomerization of the azobenzene molecule triggered by photon absorption [13]. The cyclic isomerization results in a mechanical modification of the polymer [14] and sometimes in an effective mass migration [15] that allows structuring the polymeric surface via direct illumination [16]. As massmigration occurs parallel to the polarization of the incident radiation, prefabricated microstructures can be anisotropically deformed depending on the illumination conditions $[17,18]$. In detail, we start with an array of microwells and show that the shape of the microwells can be finely controlled by exposing the film to properly polarized light. If linearly polarized radiation is used, the initial shape of microwells can be restored by turning the polarization by $90^{\circ}$. The technique presented here represents a valuable approach to tune the features of prestructured surfaces.

\section{Materials and Methods}

Sample Preparation. The azopolymer considered is an azopolyelectrolyte (poly\{1-[4-(3-carboxy-4-hydroxyphenylazo) benzenesulfonamido]-1,2-ethanediyl, sodiumsalt $\}$ ) named as PAZO (molecular weight $[\mathrm{Mw}] \approx 369,33 \mathrm{~g} / \mathrm{mol}$ ), supplied by Sigma-Aldrich. The PAZO is mixed with 


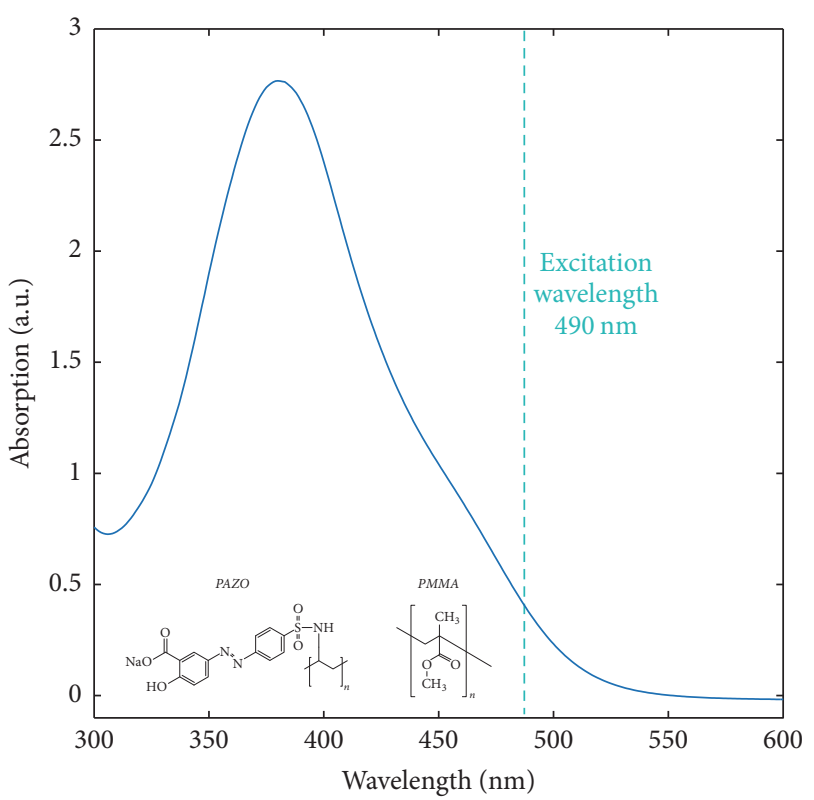

(a)

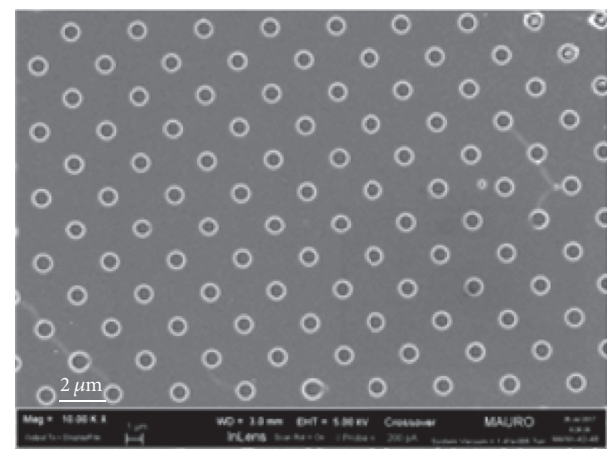

(b)

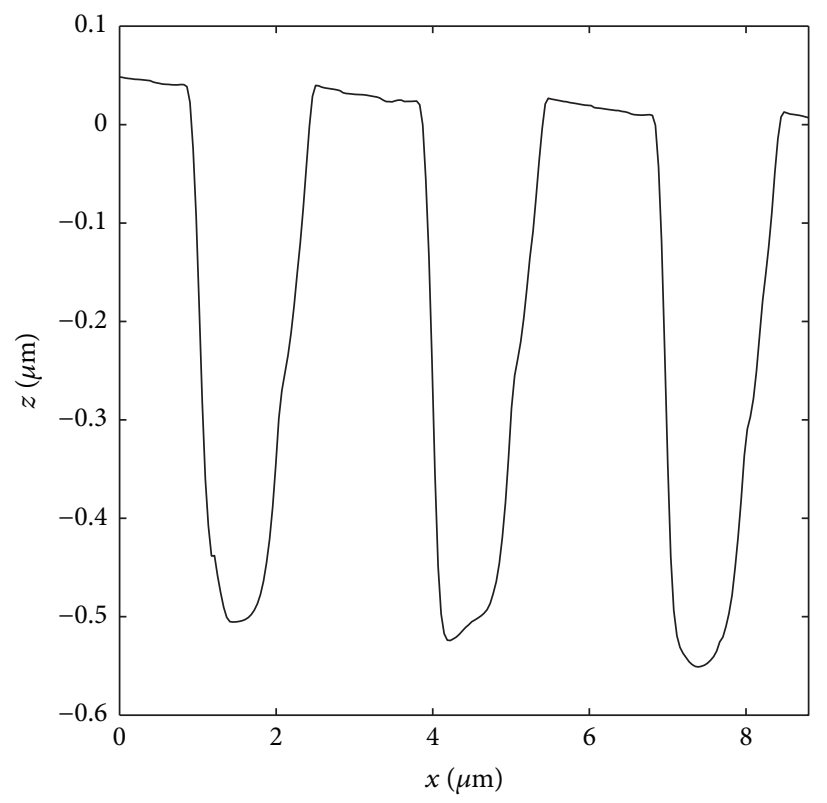

(c)

FIgure 1: (a) Absorption spectrum of the PAZO-PMMA compound in the UV-Visible range. In the inset, sketch of the chemical structure of the two polymers. (b) SEM image of the array of microwells. (c) AFM profile of the microwells.

poly-(methyl methacrylate) (PMMA), resulting in a mixture prepared as follows: PMMA powder (molecular weight $[\mathrm{Mw}] \approx 15000 \mathrm{~g} / \mathrm{mol}$ by $\left.\mathrm{GPC}^{\circledR}\right)$ is dissolved in $\mathrm{N}, \mathrm{N}$ dimethylformamide at a concentration of $4 \mathrm{wt} \%$. Nine units of PAZO $(\mathrm{Mw} \approx 369,33 \mathrm{~g} / \mathrm{mol})$, dissolved in methanol at concentration $25 \mathrm{mg} / \mathrm{mL}$, are mixed to one unit of PMMA solution and then mechanically stirred and sonicated for few minutes until a homogeneous yellow blend is formed. Figure 1(a) shows the absorption spectrum of the PMMAPAZO compound obtained and, in the inset, the molecular structure of PAZO and PMMA. We measured the glass transition temperature $(\mathrm{Tg})$ of the polymeric compound by means of Differential Scanning Calorimetry (DSC, data not shown) and we found $\mathrm{Tg}=53.9^{\circ} \mathrm{C}$ as compared to $\mathrm{Tg}=60.7^{\circ} \mathrm{C}$ of the PAZO without PMMA. An amount of $15 \mu \mathrm{l}$ of PAZO-PMMA is spin-casted onto a glass substrate, previously washed in acetone, rinsed with isopropanol, and dried with a nitrogen flow. A PDMS stamp (10:1 ratio of the prepolymer and the curing agent, provided by Sylgard ${ }^{\circledR}$ 184 , Dow Corning, cured at $60^{\circ} \mathrm{C}$ for 2 hours) is soaked in ethanol for few minutes and then gently pressed on the PAZO-PMMA film on glass. The sample is placed in a 


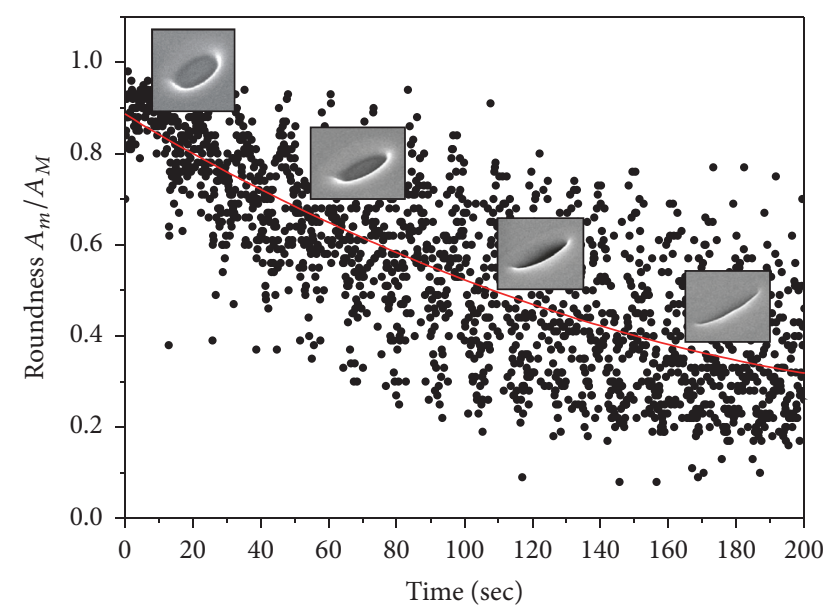

(a)

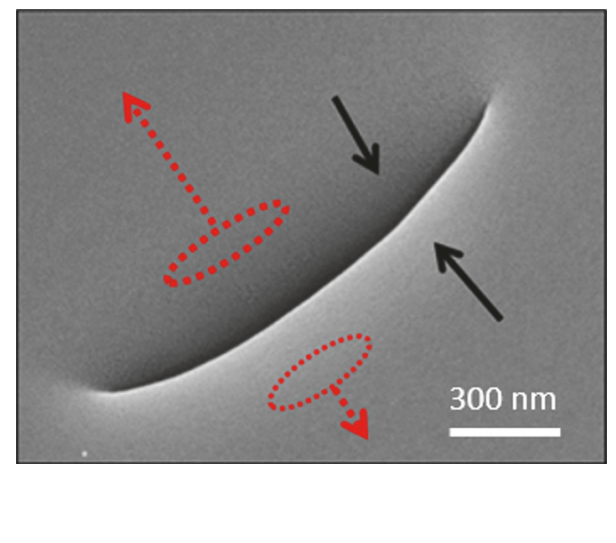

(b)

FIGURE 2: (a) Time-resolved roundness values for microwells during laser irradiation (black dots: roundness of individual wells, red line: mean roundness). In the insets, representative SEM images taken with sample tilted by $45^{\circ}$ of four different time steps. (b) SEM image (sample tilted by $45^{\circ}$ ) of a single microwell after irradiation (black arrows: the direction of the pore reduction and red arrows: direction of the mass migration of the material).

vacuum desiccator for 2 hours to enhance patterning and let the polymer blend to fill in the PDMS template. As a next step, the sample is dried in oven, until the solvent is completely evaporated ( 3 hours at $60^{\circ} \mathrm{C}$ ) before the final removal of the PDMS stamp. The SEM image in Figure 1(b) shows an array of azopolymeric microwells over a large area. The wells are circular with a diameter of $1 \mu \mathrm{m}$ arranged as a squared lattice with periodicity $\Lambda=3 \mu \mathrm{m}$. Atomic Force Microscopy analysis reveals circular wells with a diameter of $1 \mu \mathrm{m}$ arranged as a squared lattice with periodicity $\Lambda=3 \mu \mathrm{m}$. The depth, estimated by AFM analysis, is about $550 \mathrm{~nm}$, as shown in Figure 1(c).

Optical Setup. The optical setup employed in this work is described in detail in [19]. Briefly, we employ a pulsed laser (laser temporal width $\approx 10 \mathrm{ps,} \mathrm{wavelength} 490 \mathrm{~nm}$, and average intensity $\approx 50 \mathrm{~mW}$ ) circularly polarized. The laser beam is slightly focused in such a way that the spot size on the sample surface is about $20 \mu \mathrm{m}$, thus resulting in a density of power of $1.6 \mathrm{~kW} / \mathrm{cm}^{2}$. We point out that the pulsed laser has been employed because of its tunable frequency that allows for a better matching with the absorption band of PAZO (see Figure 1(a)). Due to the slow thermal relaxation time of the isomerization transition (typically in the range of hundreds of ms [15]) as compared to the repetition rate of the pulsed laser $(80 \mathrm{MHz})$, it seems reasonable to approximate the pulsed radiation to a CW light. The beam can be eventually filtered by a linear polarizer placed on a rotational stage to obtain linearly polarized light along arbitrary directions. A 100x objective (NA = 0.95) coupled with a tube lens allows imaging the sample surface on a CMOS camera (Thorlabs DCC3240M). In collection, the laser beam is filtered out by an edge filter (FEL550 from Thorlabs) along the imaging arm. In order to extract useful parameters, the images collected have been binarized by setting a proper threshold and subsequently analyzed by employing an ImageJ tool able to compute roundness and the overall area of the pores.

\section{Results and Discussion}

First, we want to investigate the effect of irradiation with linearly polarized light on the azopolymeric microstructures. In agreement with our previous experimental findings [19] and results from literature $[20,21]$, we observe a directional mass migration of the polymer along the polarization direction. For a quantitative analysis, the mean roundness parameter is evaluated for each frame as the ratio between the minor $\left(A_{m}\right)$ and the major axis $\left(A_{M}\right)$ averaged over 20 microwells within a single field-of view of the microscope. Initially, wells are almost circular, with a mean roundness of about 0.9 (Figure 2(a)). Upon linearly polarized irradiation, the wells deform into an elliptical shape (mean roundness down to 0.3 ). The SEM image in Figure 2(b), obtained keeping memory of the orientation of the sample during irradiation, reveals that the major axis of the ellipses is oriented perpendicular with respect to the polarization direction of light, confirming that the photoinduced mass migration of the material occurs along the electric field direction.

In order to show the reversibility of the deformation, we repeated the experiment with linearly polarized light. The microwells, initially showing circular shape (Figure 3(a)), turn into almost closed slits after about 220 seconds of irradiation (Figure 3(b)). Subsequently, we rotate the polarization of light by $90^{\circ}$ and we observe a gradual restore of the circular shape (Figure 3(c)). The image analysis reveals that an average roundness of about 1 is achieved after further 200 seconds of irradiation. Such reversible modification is advantageously promoted by an improved stiffness of the polymeric mixture due to the presence of a stabilizing PMMA component, as 


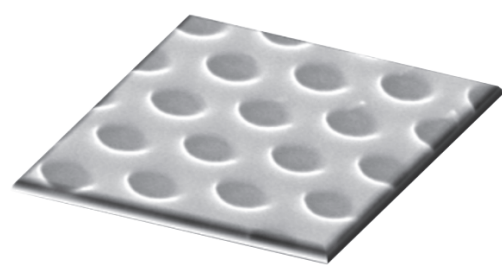

(a)

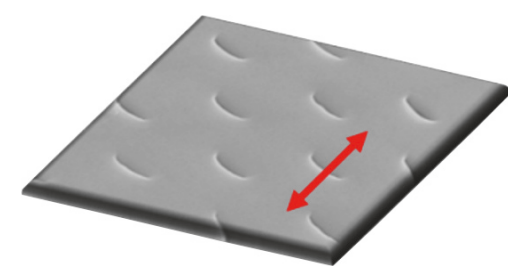

(b)

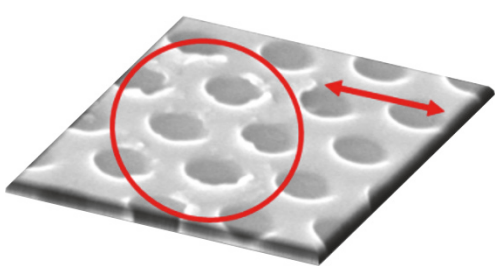

(c)

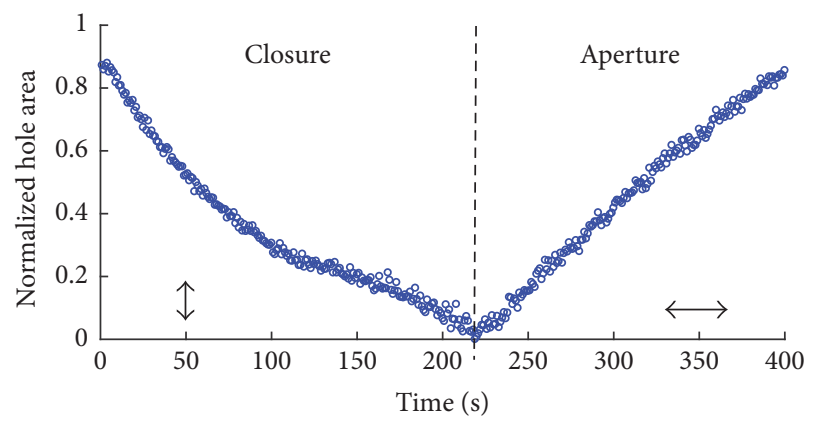

(d)

FIGURE 3: Photoreconfiguration of pristine microwells arrays by using linearly polarized light: (a) original concavity shape, (b) closed slits, and (c) restored shape. (d) Time-resolved hole area values for pores during laser irradiation with time-varying polarization states.

suggested elsewhere in other copolymer matrices containing azobenzenes [19-22]. We underline here that the role of PMMA seems to be crucial for reversibility, since with pure PAZO structures we were not able to restore the pristine shape.

In Figure 3(d) we plotted the average area of the holes versus time of irradiation. The graph shows that upon irradiation the holes area is actually reduced till a complete closing of the holes is obtained (black dashed line in the graph). Experimentally, we find that the polarization rotation by $90^{\circ}$ triggers a recovery of the microwells area. Such effect is similar to photoinduced micropillar deformation reported elsewhere [17], but the fundamental mechanism underlying the observed phenomena is not straightforward and requires further investigation.

After probing the effect of linearly polarization on the holes shape, we aim to exploit circularly polarized light to reduce the pore size eventually until complete closing. Figure 4 shows the effect of circularly polarized light on an array of microwells. The graph indicates that irradiation with circular polarization does not affect the roundness of the microstructures that is kept constant around a mean value of 0.8 . The inset in the graph shows optical images of a sequence of microwells individually addressed and irradiated with different doses (the position along the timeline corresponds to the irradiation time). The sequence shows that although the roundness is preserved, the wells tend to isotropically reduce their size until complete closure. In the case of circularly polarized light, we did not observe any reversibility related to the handedness of light polarization.

\section{Conclusions}

The presented work shows the possibility to exploit directional photofluidization to manipulate microstructures after fabrication. Starting from an array of circular microwells,

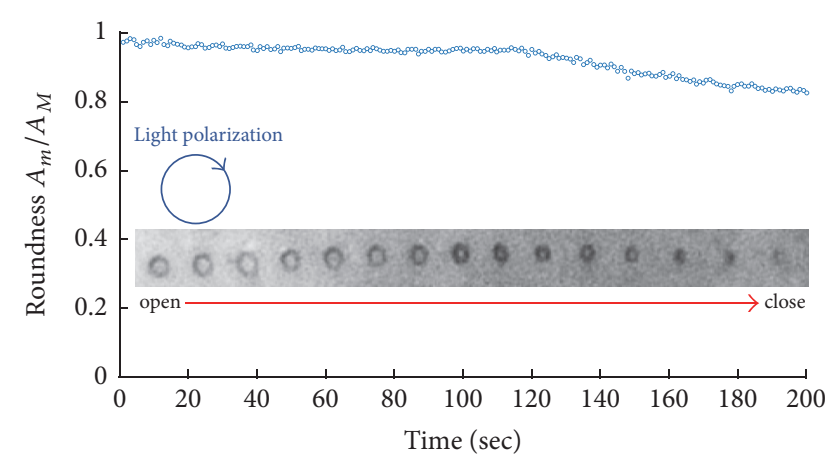

FIGURE 4: Time-resolved roundness evolution microwells during laser irradiation with circularly polarized light. In the inset, optical images of individual microwells are exposed to different light doses.

we employ linearly polarized light to change them into elliptically shaped wells and eventually into narrow slits, depending on the light dose. Alternatively, the microwells can be reduced in size without changing the roundness till an almost complete closing is reached. Tuning the morphology of surface microstructures might provide a smart engineered platform where surface properties can be engineered on demand after fabrication [19, 20]. The light-induced contraction and expansion based reshaping of polymeric microstructures shows exciting potential for several applications including microfluidics and lithography and, in the last years, is showing its potential application in biology, where cells behavior can be regulated in response to material manipulation.

\section{Conflicts of Interest}

The authors declare that there are no conflicts of interest regarding the publication of this paper. 


\section{Acknowledgments}

The authors gratefully thank Dr. Serena Ricciardi for technical support and fruitful discussions.

\section{References}

[1] A. Emoto, E. Uchida, and T. Fukuda, "Optical and physical applications of photocontrollable materials: Azobenzenecontaining and liquid crystalline polymers," Polymer, vol. 4, no. 1, pp. 150-186, 2012.

[2] Z. L. Wu, R. Wei, A. Buguin et al., "Stimuli-responsive topological change of microstructured surfaces and the resultant variations of wetting properties," ACS Applied Materials \& Interfaces, vol. 5, no. 15, pp. 7485-7491, 2013.

[3] Y. Xia and G. M. Whitesides, "Soft lithography," Angewandte Chemie International Edition, vol. 37, no. 5, pp. 550-575.

[4] M. Li, L. Chen, and S. Y. Chou, "Direct three-dimensional patterning using nanoimprint lithography," Applied Physics Letters, vol. 78, no. 21, pp. 3322-3324, 2001.

[5] D. S. Correra, L. De Boni, A. J. G. Otuka, V. Tribuzi, and C. R. Mendonça, "Two-photon polymerization fabrication of doped microstructures," in Polymerization, vol. 30, pp. 333-356, InTech, 2012.

[6] A. Arbabi, Y. Horie, M. Bagheri, and A. Faraon, "Dielectric metasurfaces for complete control of phase and polarization with subwavelength spatial resolution and high transmission," Nature Nanotechnology, vol. 10, no. 11, pp. 937-943, 2015.

[7] D. H. Kang, S. M. Kim, B. Lee, H. Yoon, and K.-Y. Suh, "Stimuliresponsive hydrogel patterns for smart microfluidics and microarrays," Analyst, vol. 138, no. 21, pp. 6230-6242, 2013.

[8] C. Rianna, A. Calabuig, M. Ventre et al., "Reversible holographic patterns on azopolymers for guiding cell adhesion and orientation," ACS Applied Materials \& Interfaces, vol. 7, no. 31, pp. 16984-16991, 2015.

[9] P. Karageorgiev, D. Neher, B. Schulz et al., "From anisotropic photo-fluidity towards nanomanipulation in the optical nearfield," Nature Materials, vol. 4, no. 9, pp. 699-703, 2005.

[10] S. Lee, H. S. Kang, and J.-K. Park, "Directional photofluidization lithography: Micro/nanostructural evolution by photofluidic motions of azobenzene materials," Advanced Materials, vol. 24, no. 16, pp. 2069-2103, 2012.

[11] S. Lee, H. S. Kang, and J.-K. Park, "High-resolution patterning of various large-area, highly ordered structural motifs by directional photofluidization lithography: Sub-30-nm line, ellipsoid, rectangle, and circle arrays," Advanced Functional Materials, vol. 21, no. 10, pp. 1770-1778, 2011.

[12] M. Saphiannikova and V. Toshchevikov, "Optical deformations of azobenzene polymers: Orientation approach vs. photofluidization concept," Journal of the Society for Information Display, vol. 23, no. 4, pp. 146-153, 2015.

[13] A. Natansohn and P. Rochon, "Photoinduced motions in azocontaining polymers," Chemical Reviews, vol. 102, no. 11, pp. 4139-4175, 2002.

[14] C. J. Barrett, J.-I. Mamiya, K. G. Yager, and T. Ikeda, "Photomechanical effects in azobenzene-containing soft materials," Soft Matter, vol. 3, no. 10, pp. 1249-1261, 2007.

[15] A. Priimagi and A. Shevchenko, "Azopolymer-based micro- and nanopatterning for photonic applications," Journal of Polymer Science Part B: Polymer Physics, vol. 52, no. 3, pp. 163-182, 2014.
[16] F. Frascella, A. Angelini, S. Ricciardi, F. Pirri, and E. Descrovi, "Surface-relief formation in azo-polyelectrolyte layers with a protective polymer coating," Optical Materials Express, vol. 6, no. 2, pp. 444-450, 2016.

[17] F. Pirani, A. Angelini, F. Frascella, R. Rizzo, S. Ricciardi, and E. Descrovi, "Light-driven reversible shaping of individual azopolymeric micro-pillars," Scientific Reports, vol. 6, Article ID 31702, 2016.

[18] J. Vapaavuori, R. H. A. Ras, M. Kaivola, C. G. Bazuin, and A. Priimagi, "From partial to complete optical erasure of azobenzene-polymer gratings: Effect of molecular weight," Journal of Materials Chemistry C, vol. 3, no. 42, pp. 11011-11016, 2015.

[19] F. Pirani, A. Angelini, S. Ricciardi, F. Frascella, and E. Descrovi, "Laser-induced anisotropic wettability on azopolymeric microstructures," Applied Physics Letters, vol. 110, no. 10, Article ID 101603, 2017.

[20] S. L. Oscurato, F. Borbone, P. Maddalena, and A. Ambrosio, "Light-driven wettability tailoring of azopolymer surfaces with reconfigured three-dimensional posts," ACS Applied Materials \& Interfaces, vol. 9, no. 35, pp. 30133-30142, 2017.

[21] A. Ambrosio, L. Marrucci, F. Borbone, A. Roviello, and P. Maddalena, "Light-induced spiral mass transport in azo-polymer films under vortex-beam illumination," Nature Communications, vol. 3, article 989, 2012.

[22] X. Zhou, Y. Du, and X. Wang, "Azo polymer janus particles and their photoinduced, symmetry-breaking deformation," ACS Macro Letters, vol. 5, no. 2, pp. 234-237, 2016. 

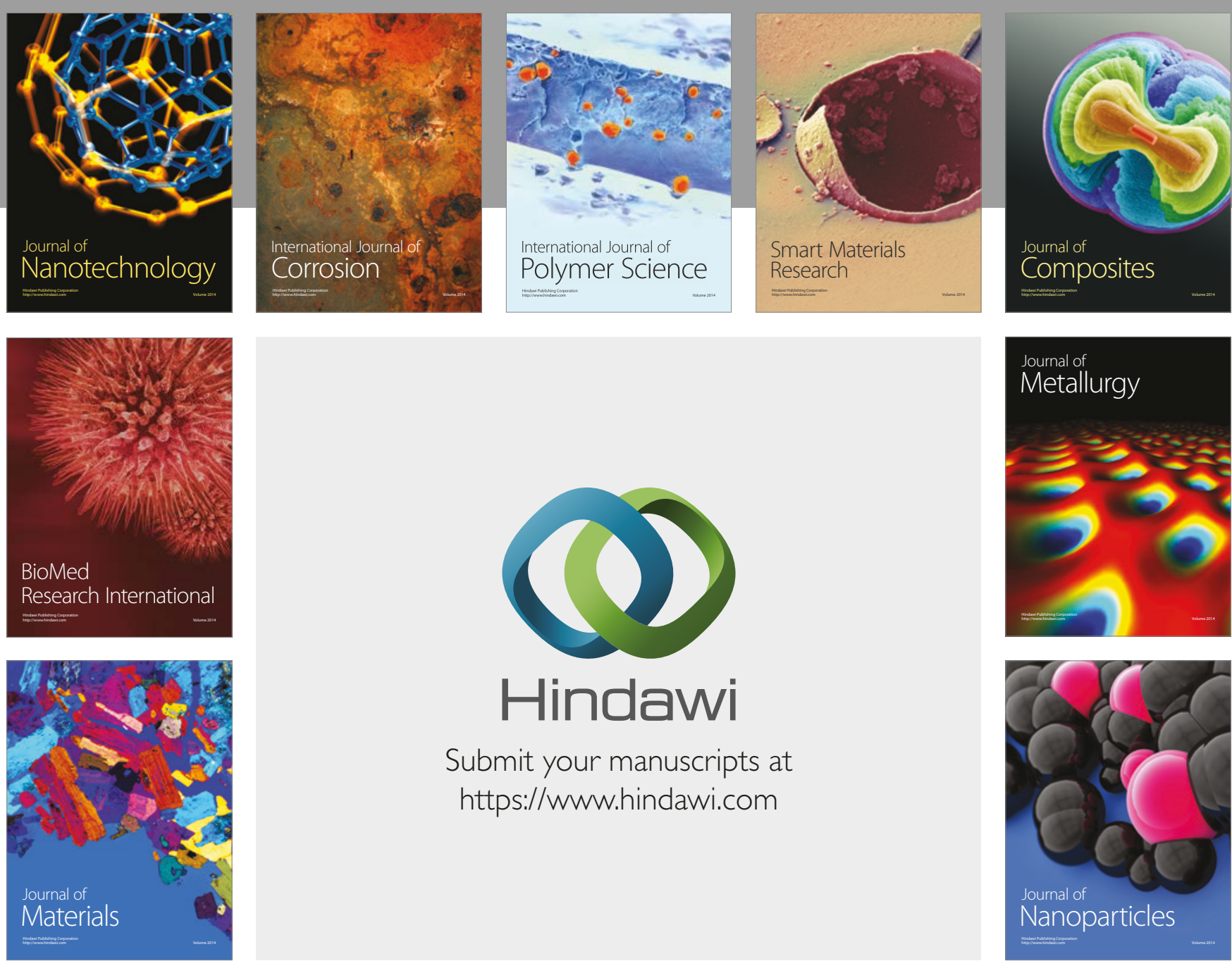

\section{Hindawi}

Submit your manuscripts at

https://www.hindawi.com
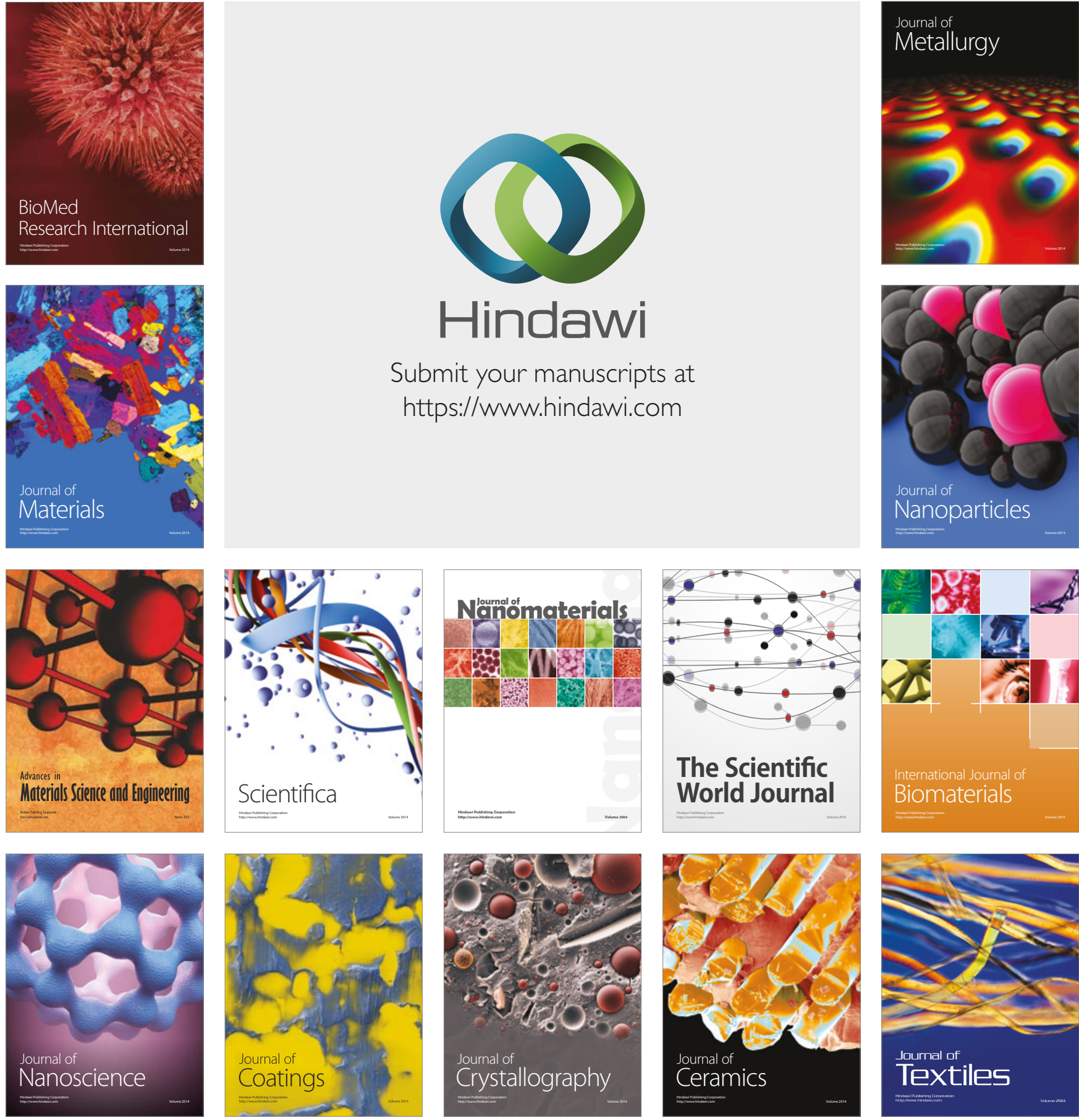

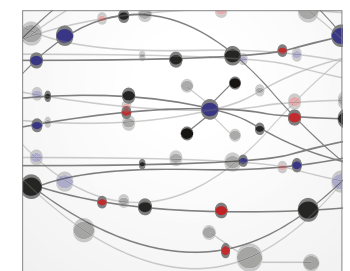

The Scientific World Journal
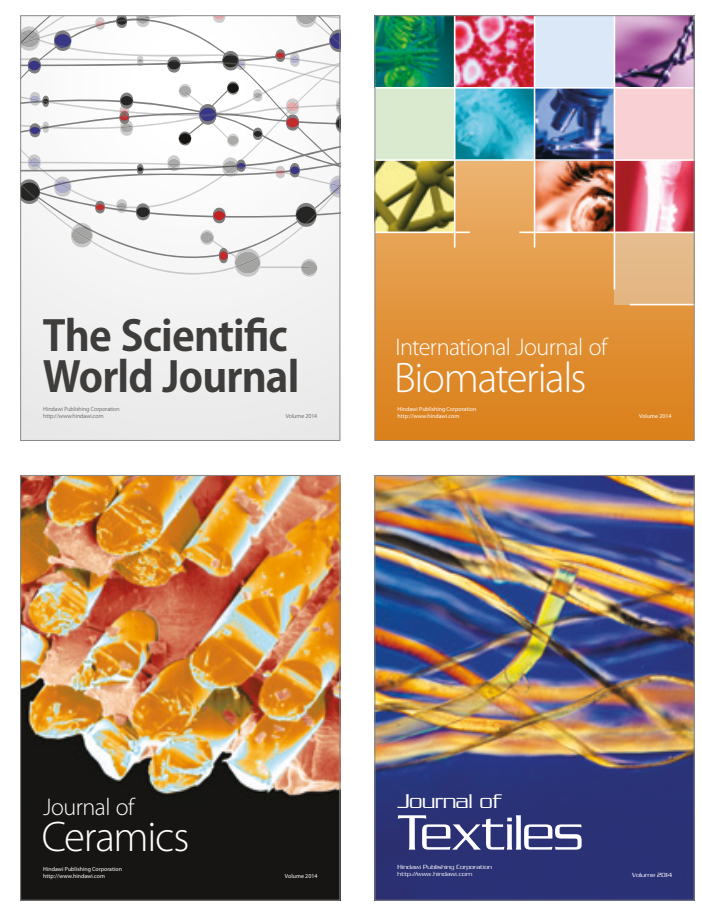